A (IM)PROVÁVE PEGADA AMBIENTAL: UMA PROVOCA ANTROPOLÓGICA SOBRE O IDEAI SUSTENTABILIDADE ENTRI "POVOS INDÍGEN 


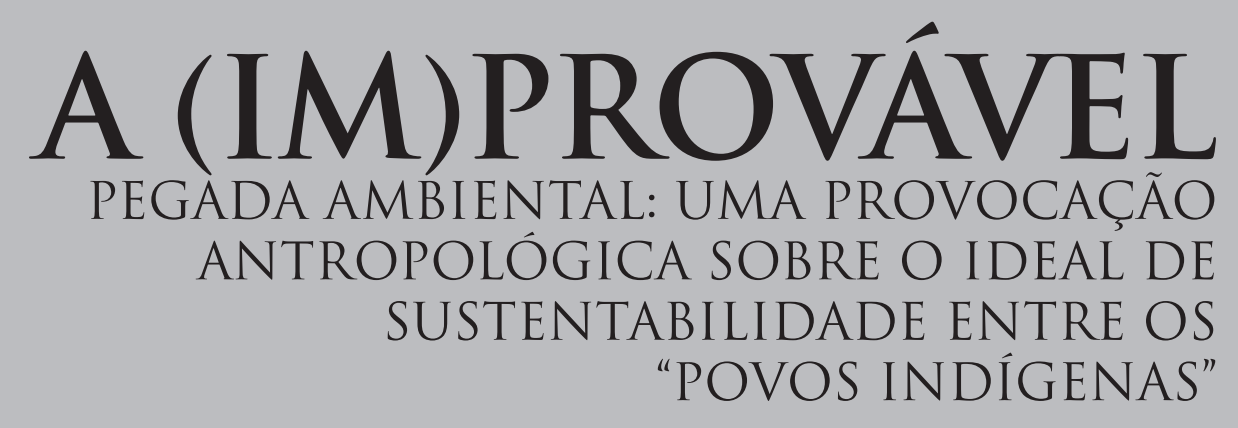

H I L T O N P. S I L V A

UNIVERSIDADE FEDERAL DO PARÁ, BRASIL 


\title{
A (IM)PROVÁVEL PEGADA AMBIENTAL: UMA PROVOCAÇÃO ANTROPOLÓGICA SOBRE O IDEAL DE SUSTENTABILIDADE ENTRE OS "POVOS INDÍGENAS"
}

\section{Resumo}

Este artigo é uma provocação à reflexão do que vai no imaginário popular e de alguns cientistas sobre os "índios" e sua relação com o ambiente natural. Visa demonstrar com exemplos brasileiros que as sociedades e culturas humanas não permanecem estáticas no tempo e no espaço, portanto, não é adequado pensar em grupos indígenas desconectando-os de sua realidade contemporânea. Não há uma sociedade "tradicional” ideal, assim como nem todos os cidadãos cosmopolitas são igualmente responsáveis pela degradação ambiental atual. Portanto, esperar que as pessoas de hoje, de qualquer grupo, permaneçam congeladas em um passado idílico, e não levar em consideração suas especificidades, fazendo generalizações simplistas sobre sua situação socioecológica, é uma temeridade acadêmica que pode ter sérias consequências para os grupos em questão e o ambiente natural.

Palavras-chave: Índios, meio ambiente, ecologia, bioantropologia, Amazônia.

\section{THE (UN) LIKELY ENVIRONMENTAL FOOTPRINT: AN ANTHROPOLOGICAL REFLECTION ABOUT THE IDEA OF SUSTAINABILITY AMONG "INDIGENOUS PEOPLES"}

\begin{abstract}
This article is a call for a reflection on the perspectives adopted by the general public and some researchers about the "Indians" and their relations with the natural environment. The objective is to demonstrate using examples from Brazil that cultures and societies are not static in time and space, therefore it is not adequate to think of indigenous groups out of connection with their contemporary reality. There is no ideal "traditional" society in the same way that all cosmopolitan citizens are not equally responsible for the current environmental degradation. Hence, to expect that people of any group today remain frozen in an idyllic past and not take into consideration their specificity, making simplistic generalizations about their socioecological situation is an academic temerity, and may have serious consequences for those groups and the environment.
\end{abstract}

Keywords: Indians, environment, ecology, bioanthropology, Amazonia. 


\section{LA (IM)PROBABLE HUELLA AMBIENTAL: UNA PROVOCACIÓN ANTROPOLÓGICA SOBRE SUSTENTABILIDAD IDEAL ENTRE LOS "PUEBLOS INDÍGENAS"}

\section{Resumen}

Este artículo es uma provocación a la reflexión de lo que sucede en el imaginario popular y de algunos científicos sobre los "indios" y su relación con el ambiente natural. Pretende demostrar con ejemplos brasileros que las sociedades y culturas humanas no permanecen estáticas en el tiempo y en el espacio, por lo que, no es apropiado pensar en grupos indígenas desconectándolos de su realidade contemporánea. No existe una sociedad "tradicional" ideal, así como no todos los ciudadanos cosmopolitas son igualmente responsables de la actual degradación ambiental. Por tanto, esperar que las personas de hoy, de cualquier grupo, permanezcan congeladas en un pasado idílico, y no tomar en cuenta sus particularidades, haciendo generalizaciones simplistas sobre su situación socioecológica, es una temeridad académica que puede acarrear serias consecuencias para los grupos interesados y el medio ambiente natural.

Palabras-clave: indios, medio ambiente, ecología, bioantropología, Amazonia.

Endereço do autor para correspondência: Programa de Pós-Graduação em Antropologia, Universidade Federal do Pará, Rua Augusto Corrêa, No 1, Cidade Universitária José da Silveira Neto, Guamá, CEP: 66075-110, Belém, PA, Brasil.E-mail: hdasilva@ufpa.br 
$\mathrm{Na}$ palestra de abertura de um importante evento de cunho socioambiental brasileiro ocorrido recentemente, um famoso economista falando sobre as relações entre populações humanas, ambiente natural, economia e sustentabilidade, reforçou seguidas vezes a ideia de que as "populações indígenas" seriam um exemplo ou, de fato, a única forma de interação sustentável de longo prazo com o ambiente natural. Um dos pesquisadores mais renomados a esposar tal perspectiva foi Darrel Posey (19472001), que trabalhou entre os Kayapó e foi um dos criadores da Etnobiologia (Posey \& Balée 1989). Mais recentemente, muitos antropólogos têm escrito, ainda que tangencialmente, sobre o tema, de forma a contribuir para alimentar essa perspectiva através de estudos de pequenos grupos étnicos nativos americanos, principalmente na Amazônia. Será que esta visão, tão amplamente disseminada na academia e em segmentos da mídia e do público em geral, não é uma idealização?

Neste artigo, proponho que a generalização sobre a "sustentabilidade" em relação a todos os grupos indígenas precisa ser analisada mais detidamente para que se possa diferenciar mito de realidade.

Não cabe aqui entrar no mérito da discussão sobre o significado de sustentabilidade, que é um debate longo e complexo (Goodland 1995, Parris \& Kates 2003, Van Bellen 2006). Para os propósitos deste artigo é suficiente utilizar o discurso mais comumente aceito pela academia de que o termo se refere simplesmente a situação de um grupo que sobrevive em uma de- terminada área ecológica sem causar nesta mudanças significativas ao longo do tempo e das suas gerações, ou seja, um grupo que tem uma baixa "pegada ecológica", como dito na referida palestra. Também não são objeto desta discussão as diversas opiniões sobre o significado de "Povos Indígenas"; o termo é usado aqui como o foi pelo palestrante, para designar historicamente os grupos que se encontravam nas Américas antes da chegada dos europeus e seus descendentes.

Como bioantropólogo, considero que o primeiro aspecto relevante dessa discussão é o temporal; ou seja: de que "Povos Indígenas" estamos tratando quando dizemos que eles são sustentáveis - os do presente ou os do passado? Uma segunda questão relevante é: de quais "Povos Indígenas" estamos falando? E um último aspecto é: sob que condições socioecológicas vivem ou viveram os indivíduos dos quais estamos falando? Veremos cada um desses aspectos a seguir para tentar, então, chegar a alguma compreensão sobre como tem se dado em realidade a interação entre os grupos genericamente chamados "Povos Indígenas" e o ambiente natural.

Sobre o recorte temporal, para se dizer que uma determinada população é sustentável é necessário estabelecer a priori se estamos falando dessa população como ela é hoje ou como ela foi no passado. Apenas para facilitar nossa análise, vamos nos concentrar nas populações ameríndias. Temos então que optar se as analisaremos da maneira como elas se encontram hoje, a par- 
tir dos impactos históricos da colonização e considerando a multiplicidade de modos de vida das diversas etnias, ou se discutiremos esses grupos a partir de informações disponíveis sobre como eles eram antes da chegada dos Europeus. Para exemplificar, vamos usar o caso do Brasil.

Atualmente há dois tipos de populações indígenas no país: os que já mantêm contato com o restante da sociedade nacional e os que se encontram afastados desse contato, no interior das florestas, notadamente na região amazônica, um dos pouquíssimos locais do planeta onde grupos com tal nível de isolamento cultural e físico do resto do mundo ocidental ainda persistem. No livro Os Povos Indigenas Isolados na Amarônia: A Luta pela Sobrevivência (Loebens \& Neves 2011), os organizadores informam que se estima a existência de 150 grupos considerados isolados no mundo, sendo que destes 127 estão na América do Sul e dentre eles 90 ficam no Brasil. Pouco se sabe sobre esses grupos, e sua existência é geralmente indicada apenas por outros "parentes" já contatados ou por vôos de reconhecimento territorial realizados pela FUNAI.

Sobre as etnias com relações já estabelecidas com o Estado, a antropologia brasileira e internacional é rica em exemplos descrevendo diversos aspectos de suas vidas e modos de interagir com o ambiente natural. Desses, sabe-se que, na maioria absoluta dos casos, os seus indicadores bio-sócio-econômicos, reconhecidos através dos dados oficiais disponíveis nos ministérios e em publicações cientí- ficas, mostram enormes disparidades em relação ao resto da sociedade nacional (Langdon 1999, Brasil 2002, Coimbra Jr. et al. 2003, Nogueira 2011, Coimbra et al., 2013). Em todos os indicadores, de mortalidade infantil a acesso a emprego, a situação dos grupos indígenas é muito mais precária que a do conjunto da população brasileira não-indígena. No entanto, é fato que, do ponto de vista do ambiente natural, nos locais onde há populações nativas o percentual de áreas menos degradadas é muito maior do que em áreas onde não há tais grupos (Ricardo 2004). Portanto, sob a ótica da sustentabilidade ambiental strictu sensu, os indígenas representam um sucesso, mas, sob a ótica de sua qualidade de vida e saúde, a situação é desoladora e a sobrevivência cultural de muitas etnias, certamente não por culpa delas, é considerada ameaçada (Brasil 2002, Tebtebba Foundation 2008, Loebens \& Neves 2011, Coimbra et al, 2013). Em relação às populações pré-históricas, aquelas existentes antes do período colonial, a arqueologia e a bioantropologia têm bastante a contribuir. Embora ainda haja muitíssimo a ser investigado, principalmente na Bacia Amazônica, há pesquisas suficientes em outros locais do mundo para que se possa traçar um perfil confiável de como viviam aquelas pessoas e como era sua relação com o ambiente natural. Os grupos que conseguiram sobreviver por mais tempo culturalmente e com pouco impacto (pegada) ambiental eram grupos pequenos, familiares, possivelmente de não muito mais do que uma centena de pessoas, ligadas por elos de parentesco, com um estilo de vida nômade ou semi-nômade (tam- 
bém chamados caçadores-coletores), com limitados recursos econômicos/ bens materiais e infra-estrutura mínima. Ou seja, uma relação muito próxima do ambiente natural, sem demérito ao nosso bem mais precioso, a cultura; mas apenas marginalmente diferente do estilo de vida dos outros primatas. É desta forma que nossos ancestrais viveram durante $99 \%$ da história evolutiva de nossa espécie (Bates \& Plog 1991, Diamond 2001, Moran 2010).

Valendo-nos de informações bioarqueológicas e etnográficas, alguns dados ilustrativos da situação dos ameríndios do passado são: uma expectativa de vida que não ultrapassava a média dos 45 anos e uma taxa de mortalidade infantil próxima a 50\%. Em uma estimativa conservadora, as mulheres tinham, em média, de seis a dez filhos durante sua vida reprodutiva, que começava por volta dos 13-14 anos, e a mortalidade materna estava na casa dos 30\%-40\%, variando de acordo com a idade da mulher. As crianças e os adultos, em sua maioria, tinham nutrição apenas suficiente para sua sobrevivência diária na maior parte do ano, sendo os infantes, as mulheres grávidas e os idosos particularmente mais vulneráveis às carências protéico-calóricas e às variações sazonais. As parasitoses eram prevalentes em todas as faixas etárias e a mortalidade causada por doenças infectocontagiosas e pragas biológicas competia em volume apenas com a morte por violência. Os grupos viviam em situação de conflitos endêmicos por territórios, áreas de caça e busca por mulheres, que causavam muitas mortes e lesões, principalmente nos homens jovens. O estupro, o infanticídio e outras formas de violência contra mulheres, crianças e grupos por eles considerados minoritários ou inferiores eram prevalentes e culturalmente sancionados. Tudo o que consideramos como direitos humanos básicos não fazia o menor sentido naquela época. Esse é o quadro que dispomos sobre a realidade dos povos ameríndios do período pré ou imediatamente póscontato (Claasen \& Joyce 1997, Milner 1999, Steckel \& Rose 2002, Staden 2010). A maioria desses povos tinha uma pegada ambiental muito reduzida, mas seria eticamente impensável atualmente admitir as situações acima relatadas, algumas das quais infelizmente continuam a ocorrer, sem pelo menos tentar sobre elas interferir, como tem sido feito através das políticas especiais de saúde existentes (Brasil 2002).

É fato que nas Américas também floresceram sociedades populacionalmente grandes e complexas antes do período colonial (Roosevelt 1999, Heckenberger et al. 2008), mas muitas já estavam a caminho do esgotamento quando ocorreram os primeiros contatos com os homens brancos; outras já haviam se extinguido milhares de anos antes e, embora haja divergência sobre a complexidade dos fenômenos ligados a queda das grandes civilizações do passado (Butzer 2012), segundo Jared Diamond em seu livro Colapso, o esgotamento ambiental seria uma das causas principais para tal (Diamond 2005).

Agora a segunda questão relevante: de quais indígenas estamos falando? É 
necessário reconhecer que há enorme diversidade entre o que se convencionou chamar de "Povos Indígenas". No passado e no presente, o ambiente natural e a cultura moldaram as relações ecológicas dos grupos humanos, criando tantas diferenças entre eles quantos sejam os locais onde estes moram, sua origem ancestral e suas formas de interpretar o mundo a sua volta. Os Kayapó e Yanomami são tão bioculturalmente diferentes quanto Islandeses e Portugueses, e achar que grupos tão díspares se relacionam com o ambiente natural da mesma forma é certamente falacioso. A União Europeia discute com frequência quem é e quem não é europeu quando decide quem dela faz parte ou não e, mesmo dentro dos países, as tensões entre os diversos grupos nacionais não são poucas pelas dificuldades em definir claramente quem é e quem não é "nativo". Então, por que insistimos na ideia de um "índio" modelo e atribuímos a ele generalidades como dizer que todos são "sustentáveis"?

Em relação a sob que condições socioecológicas vivem ou viveram os índios dos quais estamos falando, uma análise simples da literatura recente e clássica mostra que o ambiente natural tem profunda influência sobre as formas e maneiras como as pessoas (sobre)vivem. Não se trata aqui de reafirmar a Ecologia Cultural de Stewart, mas apenas de constatar que, até muito recentemente (últimos seiscentos anos), um nativo amazônida não poderia comer carne de baleia, da mesma forma que um esquimó não podia comer mandioca; um Bushman do Kalahari jamais teria o mesmo conhecimento sobre navegação oceânica que um Viking, e os encantados dos Charrua eram diferentes dos encantados dos Pimas. Da mesma forma, a base tecnológica dos diversos grupos tradicionais do continente americano era e é enormemente varia$\mathrm{da}$, influenciando diretamente a pegada ecológica daquele grupo. O impacto da chegada dos europeus nas Américas é um exemplo paradigmático de como o ambiente de origem de um grupo pode influenciar a sustentabilidade de povos diversos. Tecnologias bélicas e imunidade biológica adquiridas na ecologia do Velho Mundo dizimaram os nativos americanos e permitiram a sobreposição da cultura europeia às culturas ameríndias em poucas dezenas de anos. Por isso, não há razões que justifiquem a noção generalizada de que grupos tão ecologicamente diversos como são os ameríndios, dadas as mesmas condições infraestruturais, comportaram-se ou comportem-se da mesma maneira ("sustentável" ou não) em relação à ecologia dos locais onde vivem.

Uma influente liderança indígena, participando de um evento ao qual eu compareci, certa vez declarou em "off” que, se seu grupo tivesse motosserras no passado, hoje haveria muito menos floresta em pé. Embora essa, talvez, não represente a visão de muitos outros líderes, de fato, ninguém gosta de cortar árvore com machado de pedra, construir e viver em casas que oferecem pouca proteção em relação aos elementos naturais, ficar sujeito durante o dia a um calor abrasador e durante a noite a um frio 
de monção, ou ficar exposto diariamente a nuvens de mosquitos atrás do seu sangue. Os terreiros tradicionais de algumas aldeias Tupi mostram claramente o quanto eles apreciam a limpeza dos locais onde vivem, removendo a vegetação existente nas imediações das moradias. Isso ajuda a evitar os mosquitos e melhora a circulação de ar nas malocas. Não há evidências em contrário à perspectiva de que as áreas indígenas conservadas hoje, pelo menos em grande parte o são em função do histórico abandono social e tecnológico ao qual esses grupos têm sido submetidos pela sociedade nacional, o que reforçou sua dependência dos recursos naturais primários (a preservação dos saberes sobre as plantas medicinais e a preservação do "conhecimento tradicional" são pelo menos parcialmente resultantes disso, em virtude da falta de alternativas imediatas) e os tornou incapazes de alterar substancialmente seus ecossistemas. Na medida em que esses grupos são incorporados às tecnologias da sociedade "moderna", sua pegada ambiental invariavelmente mudará.

Qualquer grupo que utilize eletricidade e deseje bens de consumo, que tenha acesso a antibióticos e controle de natalidade, que recorra ferramentas e instrumentos metálicos e mecânicos, aprecie produtos não biodegradáveis (vêm a mente as pilhas, sacos de batatas chips e fraldas descartáveis que se acumulam por todos os cantos nas aldeias e em muitas áreas rurais), e que tenha sede de tecnologia, não é ambientalmente sustentável. Essas coisas invariavelmente levarão ao seu crescimento demográfico e aumento de demanda energética, o que está ligado ao consumo de recursos naturais e à amplificação de sua pegada ecológica (Wackernagel \& Rees 1996). A história da própria sociedade ocidental é a melhor prova desse processo (Diamond 2001).

O ideal da "sustentabilidade" dos ameríndios, dos quais os grupos pré-contato representam o modelo paradigmático (os indígenas "sustentáveis" de hoje são aqueles que mantêm características similares, ainda que muitas destas existam apenas no imaginário popular) (Diegues 2008), é fruto por um lado de um olhar idílico da sociedade ocidental, que construiu, conforme discutido por Nugent em seu livro Amaronian Caboclo Society: an essay on imvisibility and peasant economy (1993), um ideário de "natureza" e de "povos da floresta" e, por outro, das limitações demográficas e da incapacidade técnica da maioria dos grupos de alterar substancialmente o ambiente, ou seja, sua literal submissão à natureza (Adams 2000, 2003). Apenas após a revolução neolítica, com a criação da cultura agrícola (literalmente agri + cultura) e a gradual ampliação das paisagens culturais é que se consolidou, ainda que parcialmente, o domínio dos seres humanos sobre o mundo biológico (Bates \& Plog 1991). Desde então, na medida em que a capacidade de entropia dos grupos aumenta, aumenta proporcionalmente sua pegada ecológica (Wackernagel \& Rees 1996).

As elevadas taxas de mortalidade no passado mantinham o tamanho populacional em um mínimo necessário para permitir a reprodução física do 
grupo, e a tecnologia disponível para a maioria deles não permitia mudar substancialmente sua ecologia através, por exemplo, de cortes de florestas inteiras, mega construções em grandes áreas, modificação dos cursos dos rios, criação de milhões de toneladas de dejetos ou acumulação desproporcional de bens materiais. Logo, não havia condições físico-culturais para se causar grandes danos aos ecossistemas naturais, especialmente danos com reflexo em escala global.

No entanto, isso não quer dizer que os grupos indígenas atuais não se esforcem pela preservação do seu habitat, ou que não devam ser vistos como parceiros privilegiados para a construção da sustentabilidade desejada. Da mesma forma que outros grupos rurais/tradicionais, eles trabalham arduamente por sua sobrevivência e são importantes os modelos que oferecem sobre como a convivência em maior harmonia com o ambiente natural é possível. O enorme conhecimento sobre a diversidade de ambientes, de flora e de fauna que está neles depositado, assim como um milenar etnoconhecimento sobre fito e zooterápicos e a agrobiodiversidade por eles cultivada, por exemplo, representam parte fundamental da herança cultural da humanidade, que precisa ser valorizada e pode contribuir sobremaneira para a criação de uma sociedade global mais justa e igualitária.

Este artigo é uma provocação à reflexão do que vai no imaginário popular e, em parte, científico sobre os "índios", e visa a reiterar que as sociedades e as culturas humanas não são estáticas. Não há uma sociedade "tradicional" ideal, assim como nem todas as sociedades cosmopolitas são igualmente responsáveis pela degradação ambiental atual. Portanto, esperar que as pessoas de hoje, de qualquer grupo, se comportem como as de ontem, e não levar em consideração suas especificidades ao fazer generalizações é uma temeridade acadêmica, que pode trazer sérias consequências para os grupos indígenas e o ambiente natural. A antropologia, ciência do estudo dos seres humanos em todas as suas dimensões, certamente não suporta tais simplificações.

\section{NOTA}

${ }^{1} \mathrm{O}$ conceito de pegada ecológica foi criado na School of Community and Regional Planning da University of British Columbia, Canadá, por William Rees e seus colaboradores. É definido como a quantidade de terras produtivas necessárias para suportar o padrão de consumo de uma dada população indefinidamente (Wackernagel \& Rees 1996, Bicknell et al. 1998).

\section{REFERÊNCIAS}

Adams, C. 2003. The Pitfalls of synchronicity: a case study of the Caiçaras in the Atlantic Rainforest of South-eastern Brazil, in Ethnographies of Conservation. Environmentalism and the Distribution of Privilege. Edited by D. G. e E. Berglund. 1ed. London: Berghahn, p. 19-31.

Adams, C. 2000. As populações caiçaras e o mito do bom selvagem: a necessidade de uma nova abordagem interdisciplinar. Revista de Antropologia, 43(1):145-182.

Bicknell, K. B., Ball R. J., Cullen, R., Bigsby, 
H. R. 1998. New methodology for the ecological footprint with an application to the New Zealand economy. Ecological Economics 27:149-160.

Bates, D. G. \& Plog, F. 1991. Human Adaptive Strategies. New York: McGraw-Hill.

Brasil. 2002. Política Nacional de Atenção à Saúde dos Povos Indígenas. $2^{a}$ edição. Brasilia: Ministério da Saúde, Fundação Nacional de Saúde.

Butzer, K. W. 2012. Collapse, environment and society. PNAS, 109(10):3632-3639.

Claasen, C. e Joyce, R. A. (Eds.). 1997. Women in Prehistory: North America and MesoAmerica (Regendering the Past). Philadelphia: University of Pennsylvania Press.

Coimbra, C. E. A, Santos, R.V. e Escobar, A. L. 2003. Epidemiologia e Saúde dos Povos Indigenas no Brasil. Rio de Janeiro: Ed. Fiocruz/Abrasco.

Coimbra, C. E. A., Santos, R. V., Welch, J. R., Cardoso, A. M., Souza, M. C., Garnelo, L., Rassi, E., Follér, M., Horta, B. L. 2013. The First National Survey of Indigenous People's Health and Nutrition in Brazil: rationale, methodology, and overview of results. BMC Public Health, 13:52.

Diamond, J. 2001. Armas, Germes e Aço: Os Destinos das Sociedades Humanas. São Paulo: Record.

2005. Collapse: How Societies Choose to Fail or Succeed. New York: Viking Peguin.

Diegues, A. C. S. 2008. O Mito Moderno da Natureza Intocada (6a Ed.). São Paulo: NUPAUB-USP/CEC, HUCITEC.

Goodland, R. 1995. The concept of environmental sustainability. Annual Review of Ecology and Systematics, 26:1-24.

Heckenberger, M. J., Russell, J. C., Fausto, C., Toney, J. R., Schmidt, M. J., Pereira, E., Franchetto, B., Kuikuro A. 2008. PreColumbian urbanism, anthropogenic landscapes, and the future of the Amazon. Science, 321(29):1214-1217, 2008.

Langdon, E. J. 1999. Saúde e povos indígenas: Os desafios na virada do século. Trabalho apresentado no $V$ Congreso Latinoamericano de Ciencias Sociales y Medicina, 7-11 de junho, Isla de Margarita, Venezuela. Disponível em: http://www.cfh.ufsc.br/ nessi/ Margsav.htm (Acesso em 07/07/2013).

Loebens, G. F. e Neves, L. J. O. (Orgs). 2011. Povos Indígenas Isolados na Amazônia: A Luta pela Sobrevivência. Manaus: Editora da Universidade Federal do Amazonas/ CIMI, 2011.

Milner, G. R. 1999. Warfare in prehistoric and early historic eastern North America. Journal of Archaeological Research, 7(2):105-151.

Moran, E. 2010. Adaptabilidade Humana: Uma Introdução a Antropologia Ecológica. $2^{\mathrm{a}}$ ed. São Paulo Editora da Universidade de São Paulo, Editora Senac: São Paulo.

Nogueira, L. M. V. 2011. A Magnitude da Tuberculose e os Itinerários Terapêuticos dos Munduruku do Pará na Amazônia Brasileira. Tese de Doutorado, Rio de Janeiro: UFRJ / EEAN.

Nugent, S. 1993. Amazonian Caboclo Society: an essay on imvisibility and peasant economy. Oxford: Berg.

Parris, T. M. e Kates, R. W. 2003. Characterizing and measuring sustainable development. Annual Review of Environmental Resources, 28:559-586.

Posey, D. A. e Balée, W. L. (Eds.). 1989. Resource Management in Amazonia: Indigenous and Folk Strategies. (Advances in Economic Botany, 7). New York: New York Botanical Garden.

Ricardo, F. (Org.) 2004. Terras Indigenas \& Unidades de Conservação da Natureza: O Desafio das Sobreposições. São Paulo: Instituto Socioambiental.

Roosevelt, A. C. 1999. The Development of 
Prehistoric Complex Societies: Amazonia, a Tropical Forest, in Complex Polities in the Ancient Tropical World (Archaeological Papers, 9). Edited by L. J. Lucero. Washington D.C.: American Anthropological Association, p. 13-33.

Staden, H. 2010. Duas Viagens ao Brasil. Porto Alegre: L\&PM.

Steckel, R. H. e Rose, J. C. (Eds.) 2002. The Backbone of History: Health and Nutrition in the Western Hemisphere. New York: Cambridge University Press.

Tebtebba Foundation. 2008. Indicators Relevant for Indigenous Peoples: A Resource Book. Baguio City: Tebtebba Foundation.

Van Bellen, H.M. 2006. Indicadores de Sustentabilidade: Uma Análise Comparativa. $2^{\mathrm{a}}$ Ed. São Paulo: FGV.

Wackernagel, M. e Rees, W. 1996. Our Ecological Footprint: Reducing Human Impact on the Earth. Gabriola Island, BC: New Society Publishers.

Recebido em 08/07/2013.

Aprovado em 23/08/2013. 